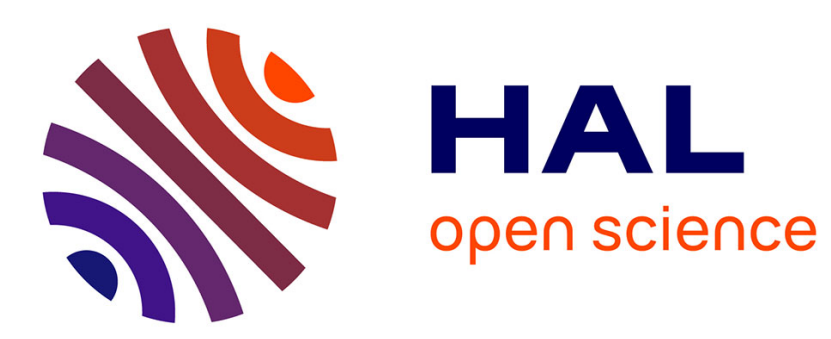

\title{
Characterizing Finger Pitch and Roll Orientation During Atomic Touch Actions
}

Alix Goguey, Géry Casiez, Daniel Vogel, Carl Gutwin

\section{To cite this version:}

Alix Goguey, Géry Casiez, Daniel Vogel, Carl Gutwin. Characterizing Finger Pitch and Roll Orientation During Atomic Touch Actions. Proceedings of the ACM Conference on Human Factors in Computing Systems (CHI 2018), Apr 2018, Montréal, Canada. pp.1-12, 10.1145/3173574.3174163 . hal-01714827

\section{HAL Id: hal-01714827 \\ https://hal.science/hal-01714827}

Submitted on 23 Feb 2018

HAL is a multi-disciplinary open access archive for the deposit and dissemination of scientific research documents, whether they are published or not. The documents may come from teaching and research institutions in France or abroad, or from public or private research centers.
L'archive ouverte pluridisciplinaire HAL, est destinée au dépôt et à la diffusion de documents scientifiques de niveau recherche, publiés ou non, émanant des établissements d'enseignement et de recherche français ou étrangers, des laboratoires publics ou privés. 


\title{
Characterizing Finger Pitch and Roll Orientation During Atomic Touch Actions
}

\author{
Alix Goguey ${ }^{1,4}$, Géry Casiez ${ }^{2,4}$, Daniel Vogel ${ }^{3}$ \& Carl Gutwin ${ }^{1}$ \\ ${ }^{1}$ University of Saskatchewan, Canada, ${ }^{2}$ Université de Lille, France, \\ ${ }^{3}$ University of Waterloo, Canada, ${ }^{4}$ Inria, France \\ alix.goguey@usask.ca, gery.casiez@univ-lille.fr, dvogel@uwaterloo.ca, gutwin@cs.usask.ca
}

\begin{abstract}
Atomic interactions in touch interfaces, like tap, drag, and flick, are well understood in terms of interaction design, but less is known about their physical performance characteristics. We carried out a study to gather baseline data about finger pitch and roll orientation during atomic touch input actions. Our results show differences in orientation and range for different fingers, hands, and actions, and we analyse the effect of tablet angle. Our data provides designers and researchers with a new resource to better understand what interactions are possible in different settings (e.g. when using the left or right hand), to design novel interaction techniques that use orientation as input (e.g. using finger tilt as an implicit mode), and to determine whether new sensing techniques are feasible (e.g. using fingerprints for identifying specific finger touches).
\end{abstract}

\section{Author Keywords}

Human factors, finger orientation, touch surface.

\section{ACM Classification Keywords}

H.5.2 Information interfaces (e.g. HCI): User interfaces

\section{INTRODUCTION}

Touch interfaces have a specific set of interactions, including tap, hold, drag, or flick, using one or multiple fingers. The capabilities and characteristics of these interactions are well known from the system's perspective (e.g. what they can be used to accomplish in an interface), but less is known about how they are carried out by users. There can be substantial variability on the user's side of the screen - for example, there are many potential ways in which a person could execute a tap, a hold, a drag, or a flick (with different fingers, or with the hand held in different postures) - and although all of these may appear identical to the touch device, there can be differences for the user.

There are several reasons why it is important for designers to understand the constraints and characteristics of the human side of touch input. This information can be used to better understand what is possible with touch - for example, certain kinds of interaction may be difficult to achieve with certain fingers, or with the non-dominant hand, or in constrained physical settings; and it may be possible to reduce error or fatigue by encouraging particular postures and orientations. Second, although touch devices currently have limited ability to sense the user's hand above the surface, these capabilities may soon be common [37], and if information about the touching hand can be sensed, it presents new design possibilities for enriching the input signal: fingers could be held at different angles to engage different implicit modes, and systems could filter touch input to reduce errors caused by inadvertent touches (e.g. a touch with a finger orientation that is well outside the normal range could be classified as accidental). Third, information about the hand can inform designers about whether certain advanced techniques are possible - for example, information about how people touch the screen can determine whether fingerprint identification is feasible (based on whether the pad of the fingertip contacts the screen), or whether fingers can be identified from their orientations alone.

An obvious first step in exploring these issues is to determine what users actually do with their hands and fingers in current interactions with touch devices. A baseline study can indicate whether there are meaningful differences in posture for different fingers, different hands, and different tasks, can determine whether some advanced interactions such as fingerprint identification are possible, and can indicate mean angles and ranges for designers who want to develop new interaction techniques using finger orientation as input. To provide this baseline information, we developed tools to test and analyse finger orientation, and carried out a study where we recorded orientation data as people performed atomic touch actions (single-finger taps, holds, swipes, and drags; and two-finger pinches and rotation gestures).

The results from our study provide both baseline information and new insights about the user's fingers above the touch screen:

- the little, ring and middle fingers of a given hand have similar pitch and roll orientations while interacting, whereas the thumb uses a complete different range.

- differences between fingers are mainly due to the difference of roll angles.

- a given finger uses different orientations as a function of the number of contacts involved in a gesture.

- there are substantial effects of tablet angle on touch orientations. 
Our data also provides us with new information about the design opportunities mentioned above:

- the relatively low pitch angles used during one-finger interactions means that it should be possible to use high pitch angles as an extra degree of freedom

- the thumb can be differentiated within the fingers of the same hand using orientation alone.

- finger identification could be enabled by fingerprint recognition if the side of the finger pad provides enough information for discrimination.

Our work makes three main contributions. First, we provide a low-cost methodology for tracking and analysing unconstrained natural finger orientation on the touch surface. Second, we provide a set of baseline results about orientation in tasks and conditions with good ecological validity for all the fingers of both hands for one setting (a flat tablet in front of the user), and additional analyses about how changing the angle of the tablet changed people's finger orientations. It helps define the ranges of used and unused orientation angles for each finger in a variety of tasks and provide insights to designers when developing interaction techniques that leverage finger orientation without interfering or changing current habits. This baseline data is made available to other researchers who wish to do further research ${ }^{1}$. Third, we provide initial explorations of several use cases that show how finger orientation information could be used to enhance interaction with future generations of touch devices.

\section{RELATED WORK}

When it comes to understand the human factor, there are at least two possible approaches. The most common one consists in studying user performance adopting the system perspective. Those studies, such as Fitts' experiments [5], allow researchers to investigate the influence of factors by examining the inputs received by a system (e.g. measuring time, error rates or accuracy). A second approach consists in adopting the user point of view by observing how we respond to a system, but also characterizing how we interact with it. In this paper we use the latter and observe how we physically use a touchscreen.

\section{Angles}

The angles used to describe the orientation of a finger are those typically used to describe airplane orientation (figure 1): the yaw (i.e. angle around the vertical axis); the pitch (i.e. angle around the lateral axis); and the roll (i.e. angle around the longitudinal axis). The yaw describes the direction at which the tip of the finger points (e.g. in a room, which wall the finger is pointing at). The pitch describes the altitude at which the tip of the finger points (e.g. in a room, is the finger pointing to the floor or the ceiling). The roll angle describes the direction that the palm of the hand faces (e.g. in a room, is the palm facing the floor or the ceiling).

\footnotetext{
${ }^{1}$ ns.inria.fr/mjolnir/fo/
}

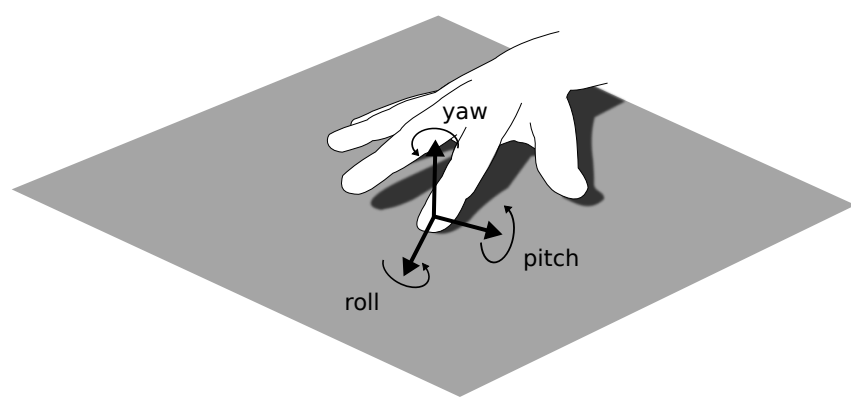

Figure 1: Angles (yaw, pitch and roll) describing a finger orientation.

\section{Studying behavior}

Knowing how people naturally interact with a system is insightful. It can guide the design of future devices in terms of ergonomics but also can lead to new degrees of freedom that can be leveraged for more expressive interactions.

Jacquier-Bret et al. observed users postures while carrying out their work on touch devices [15]. These researchers analysed which part of the body were used while executing common tasks and found that people's typical usage may increase the risk of developing musculoskeletal disorders. Their observations led them to recommend considering human motion in the development of future touch screen devices as well as exploiting their findings to better adapt the interfaces to the users capabilities. Genaro et al. studied the wrist movements of older users interacting with touch devices using a motion capture system. Using their observations, they discuss the accessibility of interactive technologies, and also encourage designers to consider the ergonomic factor to ease the adoption of touchscreens by older users [24]. Bachynskyi et al. recorded users' postures while interacting with different types of touchscreens [2]. Among other investigations, they looked at muscle activation and released a corpus to help researchers understand how we physically interact with touchscreens.

Concerning finger orientation, Mayer et al. controlled the yaw and pitch angles using $3 \mathrm{D}$ printed stands to evaluate the feasibility and comfort of touch input for stationary devices [21]. After compiling feasibility scores, they found that the non-comfortable yaw orientation zone covers $225^{\circ}$ out of $360^{\circ}$, low pitch angles are preferred when in the comfort zone while high pitch angles preferred when in the noncomfort zone. From their observations, they derived guidelines to enrich interactions. For instance, finger pitch could be used as a new input to activate contextual features (e.g. contextual menus) and close to non-comfortable orientation ranges to activate critical features (e.g. reset to factory settings). Hoggan et al. realized a similar study controlling for 2 finger rotation gestures [12]. They concluded that contralateral anti-clockwise and ipsilateral clockwise rotations are prone to more ergonomic failures.

Knowing the actual range of orientations that we use could refine Mayer et al.'s design guidelines by identifying angles that are both not yet used and also feasible. The latter could then be leveraged to enrich the touch modality. 


\section{Comparing fingers}

Although performance studies, which rely on analyzing system inputs, are primarily used to evaluate the influence of design choices (such as comparing techniques $[18,10]$ or comparing instances of a technique [9]), they are also a great tool to study real world factors (such as touchscreen orientations [26], encumbrance [25] or grasps [29]). With the rise of interaction techniques leveraging finger identification [20, 32, 8], another external factor starts to become important: our own ten fingers.

Fingers have already been compared for different tasks. McManus et al. compared the fingers through the approach of motor control by measuring the inter-tap interval (the time between two consecutive finger taps) for all fingers [23]. Colley et al. [3] and Goguey et al. [7] compared their performance as well as users' preferences in Fitts' tasks. Roy et al. also looked at their reaction times and error rates [28]. Finally, Wang et al. evaluated the precision of different fingers in a tapping task [34].

\section{Finger orientation}

Previous works have mainly focused on sensing yaw and pitch finger orientation on touch surfaces to enhance interaction techniques. Wang et al. [33] estimated the yaw angle of a finger based on the shape of the contact captured by a vision-based tabletop. For static poses, the error around the yaw estimation varies between $-5^{\circ}$ and $6^{\circ}$ ( $3^{\circ}$ on average). For dynamic poses, the error around the yaw estimation varies between $-27^{\circ}$ and $30^{\circ}$ (14 ${ }^{\circ}$ on average). In [27], Rogers et al. have a similar approach by estimating the pitch and the yaw angles from touchscreen capacitive images. They used the inferred pitch and yaw orientations to improve touch accuracy when acquiring targets. However they did not report figures comparing real finger orientations to the inferred ones. This was further exploited by Xiao et al. in [37] which not only estimated the pitch and yaw angles but also the roll angle. However, it is unclear to what extend the roll angle can be detected; this research demonstrated the use of the roll information with finger orthogonal to the screen or starting sideways and flat on the screen. For static poses, the error around the pitch estimation on a phone is $11^{\circ}$ on average. Dang et al. also use the shape of the contact to detect the yaw and pitch angles [4]. They process the contours of the contacts from the infra red based table top image. Their algorithm yields a $75 \%$ recognition rate with a tolerance of less than $5^{\circ}$ and $95 \%$ for a tolerance of less than $10^{\circ}$. Watanabe et al. estimate the force applied by a finger while contacting the screen as well as the pitch and yaw angles using a camera monitoring the light intensity emitted by the fingernail [36]. Their setup imposes a cumbersome camera mounted on the finger tip which constrains the natural finger orientation. Kratz et al. developed an algorithm capable of extracting the yaw and pitch angles by fitting a cylindrical model of the finger into a point cloud provided by a depth camera [16]. However, they acknowledge that a more complex finger model (for instance using joints) could be used to strengthen the detection. With the cylindrical model, a good orientation estimation implies a close-to-straight finger without others interfering. They reported the stability and performance of their system but no ac- curacy measurement of the orientation was reported although they acknowledge being able to compute it using a motion capture system. Mayer et al. later used this method in [22] and found a potential offset of $13.1 \%$ on the pitch angle which can only range from $0^{\circ}$ to $90^{\circ}$.

Using a vision-based system above a table, Zhang et al. estimate the yaw orientation of interacting fingers and use it to identify users in function of their position around the table top [38]. They reported the user recognition rate using their system but no accuracy measurement of the orientation was reported. Gil et al. leveraged the fact that different finger orientation yield different capacitive images [6]. They use those orientation to identify different finger on a smartwatch. However they impose a highly rolled thumb, a flat index and rolled middle finger. While the orientation is fair to assume for thumb, given the hand joints, and the index, the middle finger might not be used in a natural manner. They reported the finger recognition rates but not on the orientation retrieved.

Finally, Holz et al. introduced the use of fingerprints to increase the accuracy of touch interaction [13]. By analysing the portion of the user's fingerprint in contact and comparing it to a database of fingerprint examples, their prototype could infer the yaw, pitch and roll angles. However, even though this technological solution is promising, the recognition rate of the angles was not reported.

As measuring finger orientation is complicated, most prior work uses an approximation of the yaw and pitch angles, constrains the finger orientation, or assumes clean postures. To our knowledge, the natural pitch and roll orientations of fingers during typical touch interactions has not been investigated before.

\section{EXPERIMENT}

We conducted a within-subject design controlled experiment, to record pitch and roll angles of the 10 fingers while interacting on a touch screen.

\section{Apparatus}

To $\log$ the orientation of the fingers, we used a 0.5 " $\times 0.7$ " IMU sensor ${ }^{2}$ attached above the first phalanx using Blu Tack to avoid covering the pad of the fingertip and potentially changing participants' behavior while interacting (figure 2). The IMU was connected to an Arduino Leonardo ${ }^{3}$ plugged into a 2013 Nexus 7-inch Android tablet with a resolution of $1920 \times 1200$ pixels. A custom made Android application ran the experiment and logged the touch events as well as the orientation reported by the IMU at $50 \mathrm{~Hz}$. The tablet was fixed using a tripod at a flat orientation. Users sat on an adjustable height chair. The height was set so that the tablet was within arms reach just above the lap level.

In this paper, we thoroughly describe pitch and roll finger orientations in the screen frame of reference for all ten fingers

\footnotetext{
$\begin{array}{llll}{ }^{2} \text { Ultimate } & \text { sensor } & \text { fusion }\end{array}$

tindie.com/products/onehorse/ultimate-sensor-fusion-solution/

${ }^{3}$ Board available at \$19.80: store.arduino.cc/usa/arduino-leonardowith-headers
} 
when interacting with a flat surface. With our setup, such characterization for a non flat tablet is not possible. This state-of-the-art IMU provides reliable pitch and roll orientation in the world frame of reference, and therefore in the screen plane, using an embedded sensor fusion algorithm. However, given the nature of the movements (i.e. quick and non stable orientation over time) and the context of use (i.e. hardware in the vicinity), the relative inaccuracy of the yaw angle provided by the sensor prevents transforming the IMU orientation in a non-flat plane. The yaw angle is given by a magnetometer. Typical magnetometers allow us to keep the sensor reasonably small (i.e. smaller than the width of a finger), but come with trade off between size and accuracy. They are suited for movements that have low accelerations, but this was not the case in our study. They are also prone to interferences - such as those cause by the tablet and screen hardware (i.e. soft iron effects). Both pitch and roll angles are therefore determined using only the accelerometer and gyroscope data along with the implementation version of Madgwick's IMU and AHRS algorithms designed for invalid magnetometer measurements [17].

We considered and tested other solutions using an OptiTrack motion capture system with 4 Prime 13 cameras tracking the position and orientation of a tablet laid flat on a desk and one finger of the participant (Figure 3a). Reflective markers were attached to the tablet. One solution was to attach reflective markers onto the skin. However, vision-based systems such as this are not suitable due to frequent occlusion by the hand and infra red reflections due to the screen glass. A second approach was to shift the markers using a long $3 \mathrm{D}$ printed rigid skeleton (figure $3 \mathrm{~b}$ ) extending the orientation of the first phalanx as used in [1]. The rigid body weighed $4 \mathrm{~g}$ and had three rods of different lengths: long $(95 \mathrm{~mm})$, medium $(40 \mathrm{~mm})$ and short $(35 \mathrm{~mm}$ ) (Figure $3 \mathrm{c}$ ). It was attached such that the long rod was aligned with the first phalanx and the short rod was orthogonal to the plane defined by the fingernail. Even though lightweight minimizing tracking occlusion problems and designed to be as unobtrusive as possible, we could not ensure natural finger orientations. The choice of the IMU was therefore the best compromise: it gives us reliable absolute

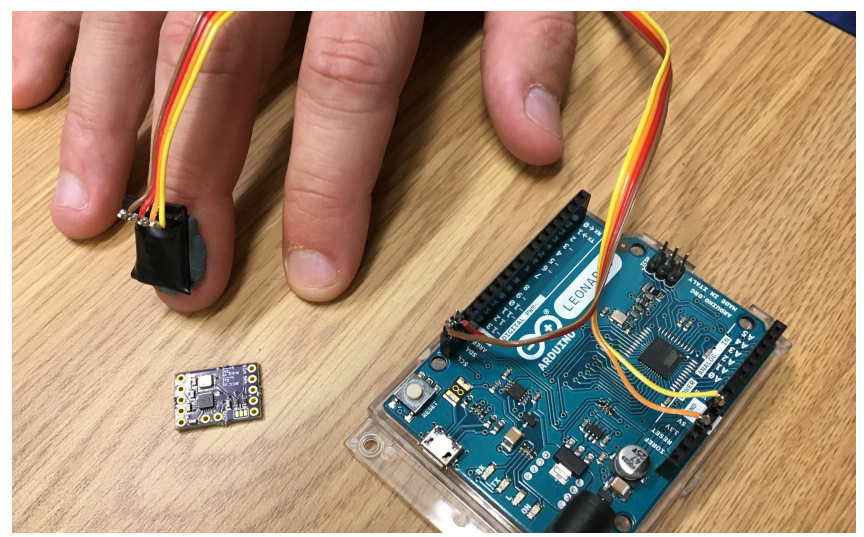

Figure 2: IMU and Arduino Leonardo board used in the experiment. The IMU was fixed on top of the first phalanx of a finger using Blu Tack.

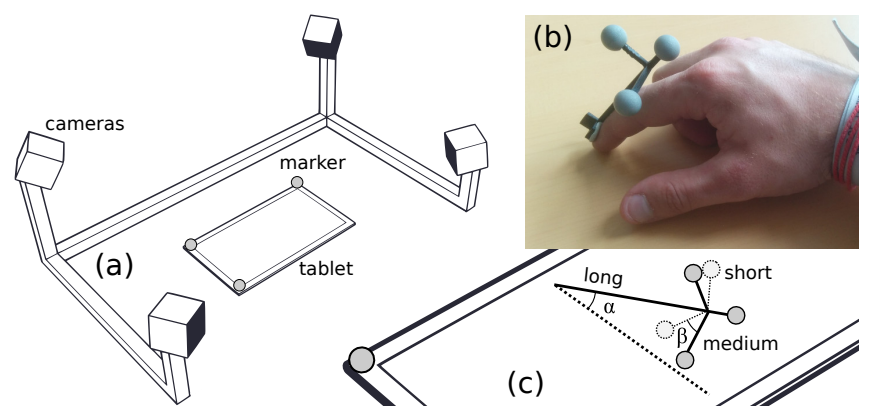

Figure 3: Motion capture solution using a rigid body attached to the fingernail: (a) tablet laid flat with $\mathbf{4}$ camera motion tracking system to track its position and orientation; (b) finger orientation was tracked using a rigid body attached to the fingernail; (c) pitch angle $\alpha$ is the angle between the long rod of the rigid body and the tablet plane, the roll angle $\beta$ is defined using the medium rod.

pitch and roll orientation but is constrained to the use of a flat tablet.

\section{Setup evaluation}

Prior to our experiment, we conducted an evaluation where we placed the IMU at known orientations using a laser-cut wood frame. For a given pitch/roll posture, we measured the orientation provided by our sensor (taking 50 samples). In between each measurement of the controlled static poses, the sensor was moved and rotated quickly away and set back to the evaluated posture. As soon as the sensor was set back, a measurement was triggered. The overall accuracy ${ }^{4}$ was $1.0^{\circ}$ for pitch and $-1.5^{\circ}$ for roll. The overall precision ${ }^{5}$ was $5.3^{\circ}$ for pitch and $8.7^{\circ}$ for roll. Table 1 shows the different precision and accuracy for the tested postures.

\section{Tasks}

Participants performed a sequence of tasks using our custom tablet application. The tasks simulated common touch interactions. In total 63 TASKS were tested. The different tasks were:

- Tap (figure 4b): pressing a circular target on the screen. 15 locations each centred on the nodes of a 5 rows by 3 column grid were tested. All the targets had the same comfortable size of $1.5 \mathrm{~cm}$ diameter.

${ }^{4}$ Distance between the mean angle and the target angle.

${ }^{5}$ Four times the standard deviation.

\begin{tabular}{rr|rr|rr}
\hline $\begin{array}{r}\text { Pitch } \\
\text { tested }\end{array}$ & $\begin{array}{r}\text { Roll } \\
\text { tested }\end{array}$ & $\begin{array}{r}\text { Pitch } \\
\text { precision }\end{array}$ & accuracy & $\begin{array}{r}\text { Roll } \\
\text { precision }\end{array}$ & accuracy \\
\hline $0^{\circ}$ & $0^{\circ}$ & $1.7^{\circ}$ & $-0.3^{\circ}$ & $0.7^{\circ}$ & $-0.1^{\circ}$ \\
$0^{\circ}$ & $45^{\circ}$ & $3.1^{\circ}$ & $-0.3^{\circ}$ & $2.7^{\circ}$ & $-0.9^{\circ}$ \\
$0^{\circ}$ & $90^{\circ}$ & $1.7^{\circ}$ & $-0.4^{\circ}$ & $2.1^{\circ}$ & $0.2^{\circ}$ \\
$45^{\circ}$ & $0^{\circ}$ & $0.9^{\circ}$ & $-1.1^{\circ}$ & $1.4^{\circ}$ & $-6.0^{\circ}$ \\
$45^{\circ}$ & $45^{\circ}$ & $3.6^{\circ}$ & $-3.6^{\circ}$ & $1.7^{\circ}$ & $-2.1^{\circ}$ \\
$90^{\circ}$ & $0^{\circ}$ & $1.6^{\circ}$ & $-0.2^{\circ}$ & $0.8^{\circ}$ & $-0.3^{\circ}$ \\
\hline all & all & $5.3^{\circ}$ & $-1.0^{\circ}$ & $8.7^{\circ}$ & $-1.5^{\circ}$ \\
\hline
\end{tabular}

Table 1: Summary of the accuracies and precisions achieved by our setup for know pitch and roll orientations. 


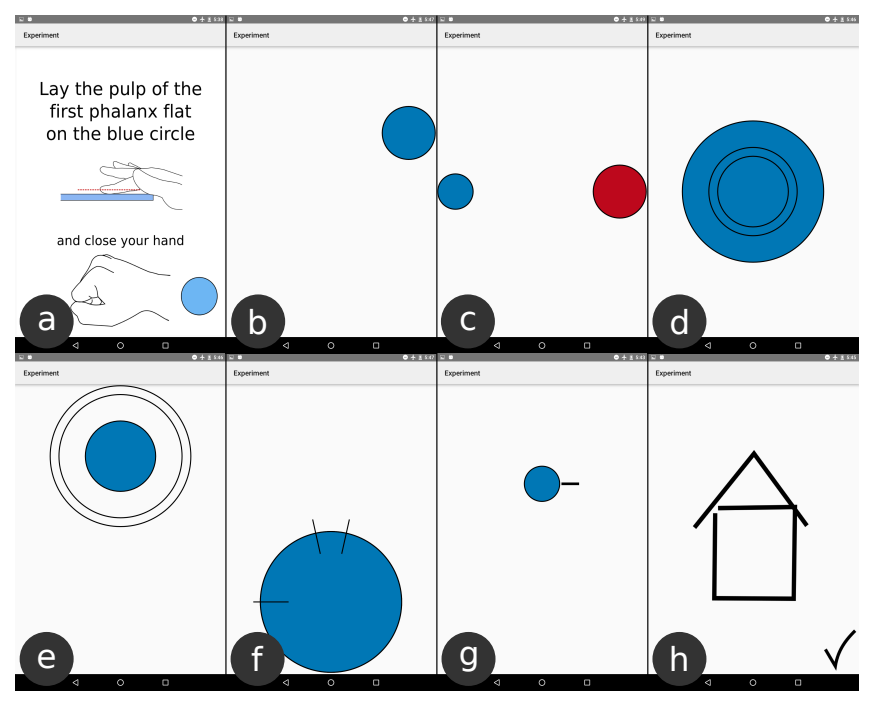

Figure 4: Different tasks explored: (a) calibration screen; (b) tap task; (c) drag task; (d) scale-down task; (e) scale-up task; (f) rotation task; (g) flick task; (h) trace task.

- Drag (figure 4c): moving a circular object inside a circular target area. 6 horizontal directions (from left to right and reverse, located at the top, middle and bottom part of the screen) and 6 vertical directions (from top to bottom and reverse, located at the left, middle and right part of the screen) were tested. All the circular objects and target areas had the same sizes of $1.6 \mathrm{~cm}$ and $2.3 \mathrm{~cm}$ diameter.

- Scale (figure 4d-e): resizing a circular object until its edge fits into a ring-shaped object. 3 scales up and 3 scales down were tested, varying only the centre position of the object (top, middle, bottom). The maximum size of the circular object was $6.3 \mathrm{~cm}$ diameter and the minimum size was $3.1 \mathrm{~cm}$ diameter. The ring object had a width of $.4 \mathrm{~cm}$.

- Rotation (figure 4f): rotating a circular object until a black mark fits between a defined aperture on the top edge of the circular object. 3 clockwise and 3 counter clockwise 90degrees rotation were tested, varying only the centre position of the object (top, middle, bottom). The angle of the aperture was 25 degrees.

- Flick (figure 4g): performing a flick gesture on a circular object. 12 flick gestures were tested: 3 starting positions of the circular object (top, middle, bottom) for each cardinal direction. The circular objects had the same comfortable size of $1.6 \mathrm{~cm}$ diameter.

- Trace (figure $4 \mathrm{~h}$ ): following a multi stroke path. 12 paths were tested: 4 different types of path (a smiley face, a house, a pig tail and a cross) centred on 3 starting position (top, middle, bottom).

Comfortable target sizes were determined according to the literature [11, 30, 35].

\section{Procedure and design}

The experimenter started by explaining the different tasks that were to be encountered during the experiment. Participants were instructed to use the selected finger for all tasks. For tasks that required two fingers, participants were free to use any other finger from the same hand. In order to observe natural orientations, we did not impose any constraint on the fingers' orientations. Participants were then showed the calibration procedure which consisted in laying the first phalanx of the imposed finger flat on a circular target and then closing the fist (figure 4a). The circular targets appeared on the left side of the screen for the left hand (close to the top left corner, middle of the left bezel or close to the bottom left corner) and on the right part of the screen for the right hand ${ }^{6}$. Participants completed each of the 63 tasks using all ten fingers. In between each task (tap and drag tasks were grouped into threes), a calibration procedure was displayed, to prevent errors from Blu Tack repositioning.

Half of the participants started with the 5 fingers of the left hand, the other half started with the fingers of the right hand. The presentation order of FINGER (thumb, index, middle, ring, little) was randomized for each hand of each participant, and the order of TASK was also randomized. Tasks which were not completed in a single movement were continued by the participant and simply resulted in more data to analyse.

Participants remained seated during the whole experiment and could take a break at any time. Performing the set of tasks took approximately 3 to 5 minutes per finger. The whole experiment lasted 45 minutes on average.

During the experiment participants were asked to report to the experimenter if the sensor was getting loose or if it interfered with their interaction. On average, this happened at most 1 or 2 times per participant. No participants reported any discomfort during the study, but a few commented that they were not used to interacting with certain fingers (such as the non-dominant-hand little finger).

\section{Participants}

We recruited 12 volunteers (mean age 27, SD 4.6, 1 female, 1 left-handed). All were daily users of multi-touch devices. Participants received an honorarium of $\$ 10$.

\section{RESULTS}

In this section we describe the pitch and roll distributions of the different fingers for all the tasks performed in the experiment. In total we gathered 449,890 data points. Each of those data points corresponds to a touch frame. As the touch input frequency is variable, we first normalized the data using a $50 \mathrm{~ms}$ period re-sampling. Given the intentional lack of instructions regarding speed, similar gestures were performed at different paces resulting in over-representation of slow movements. We therefore binned, using the mean, the re-sampled data points of all the separated touch movements, into quartiles corresponding to the quartile of the total duration of touch lifespan. After re-sampling and binning into

\footnotetext{
${ }^{6}$ Since finger morphology differs between fingers and participants, the purpose of the calibration procedure is to log the absolute pitch and roll angles of the flat phalanx which are used later to compute the actual orientation of the finger. Closing the fist avoids possible positioning bias and forces participants to use their finger naturally. Varying the position further reduces the possibility of bias.
} 


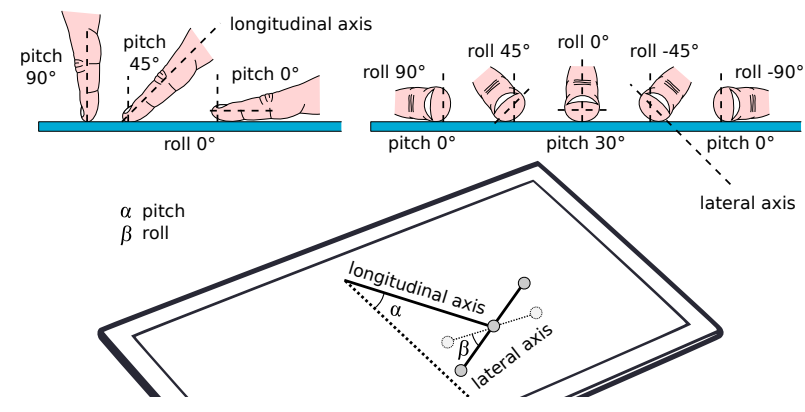

Figure 5: Definition used for the pitch and roll angles.

quartiles, 4 QUARTILES x 63 TASKS x 10 FINGERS $=2,520$ data points per participant were used for the statistics. Figure 6 however represents the $50 \mathrm{~ms}$ re-sampled data points.

All quantitative analyses are repeated measures MANOVA on both pitch and roll angles as dependent variables, using the manova command of $R$ and summarized using the Pillai-Bartlett trace. We chose MANOVAs for their robustness to the eventual lack of independence between samples (this problem can arise in the QUARTILES comparisons), as well as the fact that a violation of the multivariate normality assumption has typically little effect on the p-values [14, p138]. Furthermore, we used the Pillai-Bartlett trace which is the most conservative test and is also the most robust in cases of violation of the assumptions, at least for balanced models. Pairwise comparisons using $t$ tests with Holm adjustment method are then used when significant effects are found on each dependent variable separately. The different factors we use in our analysis are: FINGERS and QUARTILES. The latter corresponds to the different quartile of a touch lifespan: $25 \%$ corresponds to the first quartile, $50 \%$ the second quartile, $75 \%$ the third and $100 \%$ the last one.

In the different figures and tables, $T$ stands for Tap, $D$ for Drag, $S$ for Scale, $S_{d}$ for Scale-Down, $S_{u}$ for Scale-Up, $R$ for Rotation, $R_{c c}$ for Counter-Clockwise Rotation, $R_{c}$ for Clockwise Rotation, $F$ for Flick, $F_{s}$ for Flick towards south, $F_{n}$ for Flick towards north, $F_{e}$ for Flick towards east, $F_{w}$ for Flick towards west, $T r$ for Trace, $L L, L R, L M, L I$ and $L T$ represent the finger of the left hand (from little to thumb) and $R T, R I$, $R M, R R$ and $R L$ the finger of the right hand.

\section{Angle definition}

In the remainder of the paper, the pitch angle corresponds to the angle between the plane defined by the screen and the vector defined by the longitudinal axis of the finger (e.g. axis following the bone of the first phalanx). The roll is defined as $0^{\circ}$ when the lateral axis of the finger (e.g. axis traversing sideways to the first phalanx) is parallel to the tablet plane. Figure 5 illustrates the different angles and axes described.

\section{Calibration}

We found no effect of calibration position (all $\mathrm{F}<0.92$; all $\mathrm{p}>0.44)$ nor interaction between calibration position and finger (all $\mathrm{F}<0.73$; all $\mathrm{p}>0.87$ ) for the different tasks. This result suggests that the calibration procedure did not affect the orientations used by the participants during the experiment.

\begin{tabular}{r|ccc|ccc}
\hline Tasks & finger & $\begin{array}{c}\text { Min } \\
\text { mean }\end{array}$ & $95 \%$ CI & finger & $\begin{array}{c}\text { Max } \\
\text { mean }\end{array}$ & $95 \%$ CI \\
\hline $\mathrm{T}$ & $\mathrm{LL}$ & $23^{\circ}$ & {$\left[20^{\circ} ; 26^{\circ}\right]$} & $\mathrm{RT}$ & $31^{\circ}$ & {$\left[28^{\circ} ; 34^{\circ}\right]$} \\
$\mathrm{D}$ & $\mathrm{RL}$ & $24^{\circ}$ & {$\left[20^{\circ} ; 27^{\circ}\right]$} & $\mathrm{RT}$ & $37^{\circ}$ & {$\left[34^{\circ} ; 41^{\circ}\right]$} \\
$\mathrm{S}$ & $\mathrm{LT}$ & $16^{\circ}$ & {$\left[10^{\circ} ; 22^{\circ}\right]$} & $\mathrm{RI}$ & $42^{\circ}$ & {$\left[38^{\circ} ; 47^{\circ}\right]$} \\
$\mathrm{S}_{d}$ & $\mathrm{LT}$ & $14^{\circ}$ & {$\left[9^{\circ} ; 19^{\circ}\right]$} & $\mathrm{RM}$ & $42^{\circ}$ & {$\left[36^{\circ} ; 49^{\circ}\right]$} \\
$\mathrm{S}_{u}$ & $\mathrm{LT}$ & $16^{\circ}$ & {$\left[9^{\circ} ; 23^{\circ}\right]$} & $\mathrm{RI}$ & $42^{\circ}$ & {$\left[37^{\circ} ; 47^{\circ}\right]$} \\
$\mathrm{R}$ & $\mathrm{LT}$ & $23^{\circ}$ & {$\left[17^{\circ} ; 29^{\circ}\right]$} & $\mathrm{RM}$ & $50^{\circ}$ & {$\left[44^{\circ} ; 56^{\circ}\right]$} \\
$\mathrm{R}_{c c}$ & $\mathrm{LT}$ & $17^{\circ}$ & {$\left[12^{\circ} ; 22^{\circ}\right]$} & $\mathrm{LM}$ & $48^{\circ}$ & {$\left[42^{\circ} ; 54^{\circ}\right]$} \\
$\mathrm{R}_{c}$ & $\mathrm{RT}$ & $23^{\circ}$ & {$\left[18^{\circ} ; 29^{\circ}\right]$} & $\mathrm{RM}$ & $57^{\circ}$ & {$\left[49^{\circ} ; 65^{\circ}\right]$} \\
$\mathrm{F}$ & $\mathrm{LL}$ & $25^{\circ}$ & {$\left[22^{\circ} ; 27^{\circ}\right]$} & $\mathrm{RT}$ & $37^{\circ}$ & {$\left[34^{\circ} ; 40^{\circ}\right]$} \\
$\mathrm{F}_{s}$ & $\mathrm{RL}$ & $32^{\circ}$ & {$\left[29^{\circ} ; 36^{\circ}\right]$} & $\mathrm{LM}$ & $42^{\circ}$ & {$\left[38^{\circ} ; 45^{\circ}\right]$} \\
$\mathrm{F}_{n}$ & $\mathrm{LL}$ & $24^{\circ}$ & {$\left[20^{\circ} ; 28^{\circ}\right]$} & $\mathrm{RM}$ & $33^{\circ}$ & {$\left[28^{\circ} ; 38^{\circ}\right]$} \\
$\mathrm{F}_{e}$ & $\mathrm{LL}$ & $19^{\circ}$ & {$\left[16^{\circ} ; 22^{\circ}\right]$} & $\mathrm{RT}$ & $41^{\circ}$ & {$\left[37^{\circ} ; 46^{\circ}\right]$} \\
$\mathrm{F}_{w}$ & $\mathrm{RL}$ & $21^{\circ}$ & {$\left[17^{\circ} ; 25^{\circ}\right]$} & $\mathrm{LM}$ & $36^{\circ}$ & {$\left[32^{\circ} ; 41^{\circ}\right]$} \\
$\mathrm{Tr}$ & $\mathrm{LL}$ & $23^{\circ}$ & {$\left[21^{\circ} ; 25^{\circ}\right]$} & $\mathrm{RT}$ & $39^{\circ}$ & {$\left[36^{\circ} ; 42^{\circ}\right]$} \\
\hline
\end{tabular}

Table 2: Summary of the minimum and maximum mean pitch angle and corresponding $95 \%$ CI, for each task. Fingers for which minimum and maximum values were achieved are indicated.

\section{Pitch and roll distributions}

Figure 6 shows the distributions of the pitch and roll angles for all the data points gathered for each finger and each task. In table 3, we summarize the results of the statistical tests performed for each task on pitch and roll as dependent variables and FINGERS and QUARTILE as factors.

We found a significant main effect of FINGER for the different tasks. Post-hoc tests reveal numerous significant differences between fingers for roll (table 5) and pitch (table 6). The differences seem to be mainly due to the roll angle. Table 2 summarizes the minimum and maximum mean pitch angles, as well as the $95 \%$ confidence interval, for each task and for which finger those were achieved. We also report the equivalent for the roll angle in table 4 . Thumbs are without surprise the fingers with the most extreme means. According to figure 6 and table 5, within each hand, the little, ring and middle fingers are mostly similar, the index finger is similar to the middle finger and the thumb is apart (except for the right thumb and little finger). The differences between fingers according to the pitch variables are less marked. Those differences mostly come from actions inducing a high range of motion like rotations or scalings.

These different behaviours can be explained by hand morphology. The index finger is more dexterous and is often used with a closed-hand "pointing" pose, enabling a range of motion roll centered on the table surface with little interference from other fingers. The middle, ring and little finger have less dexterity and are typically used with an open hand making it hard to interact with the side part of the finger facing the thumb without having other fingers interfere with erroneous tablet contacts. It results in a slight roll shift in the distributions (toward negative roll values for the left hand and positive roll values for the right hand). Since the thumb is opposable to the other fingers, there is a symmetrical problem, resulting in a symmetrical distribution (with the roll values shifted toward positive values for the left hand and negative values for the right hand). 


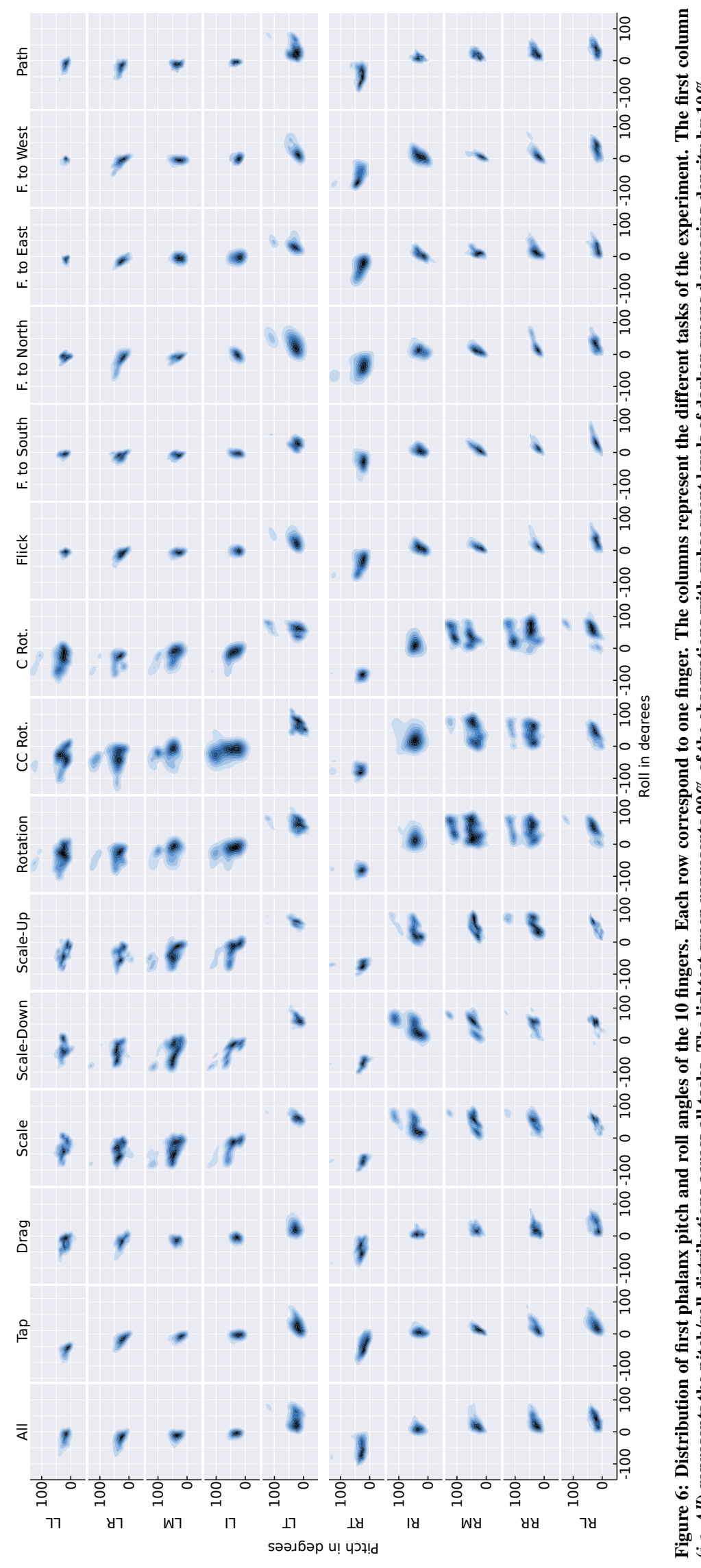

\begin{tabular}{|c|c|c|c|}
\hline & & & \\
\hline 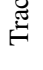 & $\vec{\sim} \circlearrowleft \overrightarrow{\mathrm{V}}$ & Tढ。 & $\stackrel{m}{i}: \dot{\vec{v}}$ \\
\hline 竎 & 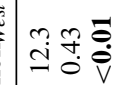 & : : : & $9 \stackrel{\infty}{-} \stackrel{\infty}{0} \stackrel{0}{0}$ \\
\hline 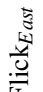 & অ̆ & 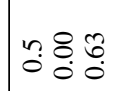 & $\stackrel{\infty}{-\infty} \stackrel{\infty}{\circ} \stackrel{0}{0}$ \\
\hline 竞 & 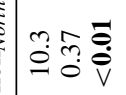 & $0.8 \stackrel{\infty}{\circ}$ & $\underset{\sim}{n} \frac{0}{0} \overline{\dot{0}}$ \\
\hline 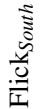 & 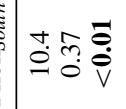 & 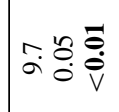 & 동 \\
\hline$\frac{\breve{.}}{\underline{I}}$ & $\begin{array}{l}\stackrel{0}{g} \overrightarrow{0} \\
\dot{I} \\
\dot{\sigma}\end{array}$ & m: & $\stackrel{\infty}{\infty} \stackrel{0}{0} \stackrel{0}{0}$ \\
\hline 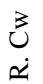 & 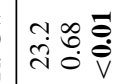 & 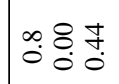 & $\stackrel{n}{i} \stackrel{0}{0} \overline{\dot{\theta}}$ \\
\hline 己 & 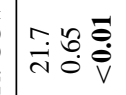 & $m$ & $\stackrel{0}{0} \stackrel{0}{0}$ \\
\hline 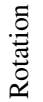 & 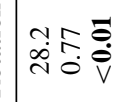 & 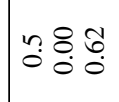 & 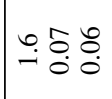 \\
\hline $\begin{array}{l}\vec{s} \\
\dot{s}\end{array}$ & 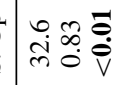 & - & 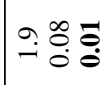 \\
\hline @̃ & 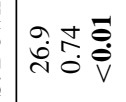 & 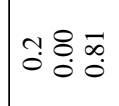 & ؟ִ \\
\hline $\begin{array}{l}\frac{0}{\tilde{J}} \\
\text { nू }\end{array}$ & 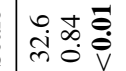 & : & Ұ: \\
\hline 竎 & 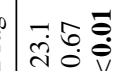 & t. & mo: \\
\hline 余 & 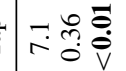 & 융 & $\stackrel{+}{-} \stackrel{\infty}{0}=$ \\
\hline & 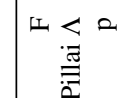 & 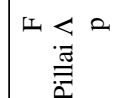 & 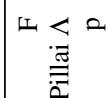 \\
\hline & 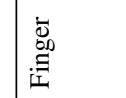 & 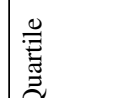 & 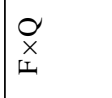 \\
\hline
\end{tabular}




\begin{tabular}{r|ccc|ccc}
\hline Tasks & finger & $\begin{array}{c}\text { Min } \\
\text { mean }\end{array}$ & $95 \%$ CI & finger & $\begin{array}{c}\text { Max } \\
\text { mean }\end{array}$ & $95 \%$ CI \\
\hline $\mathrm{T}$ & RT & $-33^{\circ}$ & {$\left[-38^{\circ} ;-28^{\circ}\right]$} & LT & $37^{\circ}$ & {$\left[33^{\circ} ; 40^{\circ}\right]$} \\
$\mathrm{D}$ & $\mathrm{RT}$ & $-36^{\circ}$ & {$\left[-40^{\circ} ;-32^{\circ}\right]$} & LT & $39^{\circ}$ & {$\left[36^{\circ} ; 42^{\circ}\right]$} \\
$\mathrm{S}$ & $\mathrm{RT}$ & $-66^{\circ}$ & {$\left[-69^{\circ} ;-62^{\circ}\right]$} & LT & $68^{\circ}$ & {$\left[65^{\circ} ; 71^{\circ}\right]$} \\
$\mathrm{S}_{d}$ & $\mathrm{RT}$ & $-67^{\circ}$ & {$\left[-71^{\circ} ;-63^{\circ}\right]$} & LT & $70^{\circ}$ & {$\left[67^{\circ} ; 73^{\circ}\right]$} \\
$\mathrm{S}_{u}$ & $\mathrm{RT}$ & $-65^{\circ}$ & {$\left[-69^{\circ} ;-61^{\circ}\right]$} & LT & $67^{\circ}$ & {$\left[63^{\circ} ; 70^{\circ}\right]$} \\
$\mathrm{R}$ & $\mathrm{RT}$ & $-71^{\circ}$ & {$\left[-74^{\circ} ;-67^{\circ}\right]$} & LT & $71^{\circ}$ & {$\left[68^{\circ} ; 74^{\circ}\right]$} \\
$\mathrm{R}_{c c}$ & $\mathrm{RT}$ & $-68^{\circ}$ & {$\left[-73^{\circ} ;-62^{\circ}\right]$} & LT & $77^{\circ}$ & {$\left[73^{\circ} ; 80^{\circ}\right]$} \\
$\mathrm{R}_{c c}$ & $\mathrm{RT}$ & $-73^{\circ}$ & {$\left[-78^{\circ} ;-68^{\circ}\right]$} & LT & $67^{\circ}$ & {$\left[64^{\circ} ; 71^{\circ}\right]$} \\
$\mathrm{F}$ & $\mathrm{RT}$ & $-36^{\circ}$ & {$\left[-41^{\circ} ;-32^{\circ}\right]$} & LT & $40^{\circ}$ & {$\left[37^{\circ} ; 44^{\circ}\right]$} \\
$\mathrm{F}_{s}$ & $\mathrm{RT}$ & $-27^{\circ}$ & {$\left[-32^{\circ} ;-21^{\circ}\right]$} & LT & $35^{\circ}$ & {$\left[30^{\circ} ; 39^{\circ}\right]$} \\
$\mathrm{F}_{n}$ & $\mathrm{RT}$ & $-36^{\circ}$ & {$\left[-42^{\circ} ;-31^{\circ}\right]$} & LT & $43^{\circ}$ & {$\left[39^{\circ} ; 47^{\circ}\right]$} \\
$\mathrm{F}_{e}$ & $\mathrm{RT}$ & $-33^{\circ}$ & {$\left[-40^{\circ} ;-27^{\circ}\right]$} & LT & $46^{\circ}$ & {$\left[42^{\circ} ; 50^{\circ}\right]$} \\
$\mathrm{F}_{w}$ & $\mathrm{RT}$ & $-44^{\circ}$ & {$\left[-49^{\circ} ;-39^{\circ}\right]$} & LT & $39^{\circ}$ & {$\left[33^{\circ} ; 44^{\circ}\right]$} \\
$\mathrm{Tr}$ & $\mathrm{RT}$ & $-35^{\circ}$ & {$\left[-39^{\circ} ;-31^{\circ}\right]$} & LT & $43^{\circ}$ & {$\left[39^{\circ} ; 47^{\circ}\right]$} \\
\hline
\end{tabular}

Table 4: Summary of the minimum and maximum mean roll angle and corresponding $95 \%$ CI, for each task. Fingers for which minimum and maximum values were achieved are indicated.

\section{Time distributions}

We found no significant effect of QUARTILES (second row of table 6). However we found significant interactions between FINGERS and QUARTILES for some of the tasks (third row of table 6). Post-hoc analysis focused on each finger (with no adjustment method) revealed differences between: the first and third quartile on roll for the left thumb for the counterclockwise rotation task $(\mathrm{p}<0.04)$; the last quartile and all the others on roll for the left ring (all $\mathrm{p}<0.04$ ), the left middle (all $\mathrm{p}<0.01$ ) and the left index finger (all $\mathrm{p}<0.02$ ) for the flick toward South task; and the first and last quartile on roll for the right thumb for the flick toward East task $(\mathrm{p}<0.04)$. Those results suggest a certain consistency within a single touch in the pitch/roll orientation while interacting. However, given our experimental design, it could also be due to the short nature of the touch lifespans.

\section{Task distributions}

We ran similar analysis, for each finger with TASKS and QUARTILES as factors.

We found a significant main effects of TASK for all the different fingers (all $\mathrm{F}>3.4$; all $\mathrm{p}<0.01$ ). After gathering post-hoc tests for pitch and roll (see table 7 ) analysis revealed that tapping, dragging and flicking distributions are similar for most of the fingers and significantly different from both scaling and rotation distributions. Scaling and rotation distributions are also significantly different for the majority of fingers. We found no effect of QUARTILE nor interactions between TASK and QUARTILE (all $\mathrm{F}<1.5$; all $\mathrm{p}>0.22$ ). Those results suggest that finger orientations differ in function of the number of fingers interacting at the same time.

\section{FOLLOW UP EXPERIMENT}

In order to test the influence of the tablet orientation, we conducted a follow-up study using the same apparatus and procedure as the main experiment. In this study, we tested two tablet orientations: tilted at $15^{\circ}$ and tilted at $30^{\circ}$. For each conditions, we ran 8 new volunteers $\left(15^{\circ}\right.$ : mean age $25.8, \mathrm{SD}$ 5.4, 3 females, 2 left-handed; $30^{\circ}$ : mean age 27.6, SD 9, 4 females, 1 left-handed). All participants were also daily users of multi-touch devices as in the first experiment.

In the following, we analyse the pitch and roll angles in the world frame of reference. We used the same statistical methods as the previous experiment. All quantitative analyses are repeated measures MANOVA on both pitch and roll angles as dependent variables summarized using the Pillai trace. Pairwise comparisons using t tests with Holm adjustment method are then used when significant effects are found on each dependent variables separately. We tested ORIENTATIONS, corresponding to the different tilts of the tablet, as a between subject factor.

\section{Influence of tablet orientation}

For each pair of task and finger, we found a significant main effect of ORIENTATION on both pitch and roll dependent variables (all $\mathrm{F}>6.2$; all Pillai $\Lambda>0.22$; all $\mathrm{p}<0.01$ ). Posthoc analysis revealed differences between all orientations for each pair of task and finger (all $\mathrm{p}<0.05)$ except between tilt $15^{\circ}$ and $30^{\circ}$ for the right thumb during rotations tasks including clockwise and counter-clockwise rotations alone (all $\mathrm{p}>0.17)$. This result suggests that participants adapted their finger orientation as a function of the tablet tilt. One could hypothesize that we use similar finger orientations in the screen frame of reference to those used when interacting with a flat tablet. If so, the previous descriptions of finger orientations on flat screen as well as their derived design insights could still stand. However, further experiments are necessary to confirm or reject this hypothesis.

\section{Range of orientations}

We also looked at the bounding boxes (length of the pitch and roll ranges in the world frame of reference) of the distribution to assess if there are differences in terms of range of orientations. The results generally follow the same trend: for the vast majority of pairs task $\times$ finger, except while tapping, flicking South, East and West, the change in the tablet tilt has a significant impact on the range of orientation. Post-hoc tests and analysis of the mean bounding box (table 8) revealed the following trend: when there are differences, it appears that the more the tablet is tilted, the smaller the range of orientation used (except for the right thumb during scaling tasks). The latter observation would suggest that (except while tapping, flicking South, East and West) participants used more consistent finger orientations when the tablet was pitched at $15^{\circ}$ and $30^{\circ}$ compared to the tablet laid flat. In these cases, the range of orientations described in the flat tablet conditions are different. However, one can hypothesize that the greater consistency of finger orientations can be centred around the mean orientation of the flat tablet distributions. If so, some design insights (e.g. using high pitch as a secondary touch input) could still stand. Again, further analysis are needed to test those hypotheses.

\section{DISCUSSION}

Using figure 6 and table 2, we can observe that the orientations used while interacting are relatively low pitched on average. Following Mayer et al. design guidelines [21], high pitched values can be comfortably used to enable secondary 


\begin{tabular}{|c|c|c|c|c|c|c|c|c|c|}
\hline & LL & LR & LM & LI & LT & RT & RI & $\mathrm{RM}$ & RR \\
\hline LL & - & - & - & - & - & - & - & - & - \\
\hline LR & & - & - & - & - & - & - & - & - \\
\hline LM & $\mathrm{R}_{\text {all }}, \mathrm{Tr}$ & $\mathrm{R}_{\text {all }}, \mathrm{F}, \mathrm{F}_{n}, \mathrm{Tr}$ & - & - & - & - & - & - & - \\
\hline LI & $\mathrm{D}, \mathrm{R}, \mathrm{R}_{c c}, \mathrm{~F}, \mathrm{~F}_{e}, \mathrm{~F}_{w}, \mathrm{Tr}$ & all but $\mathrm{T}, \mathrm{S}, \mathrm{S}_{d}$ & $\mathrm{D}$ & - & - & - & - & - & - \\
\hline LT & all tasks & all tasks & all tasks & all tasks & - & - & - & - & - \\
\hline $\mathrm{RT}$ & all tasks & all tasks & all tasks & all tasks & all tasks & - & - & - & - \\
\hline RI & all tasks & all tasks & all but $\mathrm{F}_{w}$ & all but $\mathrm{F}_{e}, \mathrm{~F}_{w}$ & all tasks & all tasks & - & - & - \\
\hline $\mathrm{RM}$ & all tasks & all tasks & all tasks & all but $\mathrm{F}_{w}$ & all but $\mathrm{F}_{n}$ & all tasks & $\mathrm{D}, \mathrm{R}, \mathrm{F}, \mathrm{F}_{w}, \mathrm{Tr}$ & - & - \\
\hline RR & all tasks & all tasks & all tasks & all tasks & all but $\mathrm{T}, \mathrm{S}_{u}, \mathrm{R}_{c}, \mathrm{~F}_{s}, \mathrm{~F}_{n}$ & all tasks & all but $\mathrm{T}, \mathrm{S}_{d}, \mathrm{R}_{c c}$ & & - \\
\hline $\mathrm{RL}$ & all tasks & all tasks & all tasks & all tasks & $\mathrm{S}_{d}, \mathrm{R}, \mathrm{R}_{c c}, \mathrm{~F}_{e}$ & all tasks & all but $\mathrm{R}_{c c}$ & all but $\mathrm{S}_{\text {all }}, \mathrm{R}, \mathrm{R}_{c c}$ & $\mathrm{~T}, \mathrm{D}, \mathrm{F}, \mathrm{F}_{s}, \mathrm{Tr}$ \\
\hline
\end{tabular}

Table 5: Summary of the post-hoc analysis on Roll for the FINGER factor. The cell of the table indicates the tasks on which two fingers (first row and first column) differ. All $\mathbf{p}<\mathbf{0 . 0 5}$.

\begin{tabular}{|c|c|c|c|c|c|c|c|c|c|}
\hline & LL & LR & LM & LI & LT & RT & $\mathrm{RI}$ & $\mathrm{RM}$ & $\mathrm{RR}$ \\
\hline $\mathrm{LL}$ & - & - & - & - & - & - & - & - & - \\
\hline LR & $\mathrm{D}, \mathrm{S}_{d}, \mathrm{R}, \mathrm{R}_{c c}, \mathrm{~F}, \mathrm{~F}_{e}, \mathrm{Tr}$ & - & - & - & - & - & - & - & - \\
\hline LM & all but $\mathrm{T}, \mathrm{R}_{c}, \mathrm{~F}_{e}$ & $\mathrm{D}, \mathrm{S}, \mathrm{S}_{u}$ & - & - & - & - & - & - & - \\
\hline LI & $\mathrm{D}, \mathrm{S}_{\text {all }}, \mathrm{Tr}$ & $\mathrm{S}, \mathrm{S}_{u}$ & $\mathrm{D}, \mathrm{F}_{n}$ & - & - & - & - & - & - \\
\hline LT & $\mathrm{D}, \mathrm{F}, \mathrm{F}_{e}, \mathrm{~F}_{w}, \mathrm{Tr}$ & $\mathrm{S}_{d}, \mathrm{R}, \mathrm{R}_{c c}$ & $\mathrm{D}, \mathrm{S}_{a l l}, \mathrm{R}, \mathrm{R}_{c c}, \mathrm{~F}_{n}$ & $\mathrm{~S}_{a l l}, \mathrm{R}_{c c}, \mathrm{~F}_{e}$ & - & - & - & - & - \\
\hline RT & $\mathrm{D}, \mathrm{F}, \mathrm{F}_{e}, \mathrm{~F}_{w}, \mathrm{Tr}$ & $\mathrm{S}_{d}, \mathrm{R}, \mathrm{R}_{c}, \mathrm{~F}_{w}$ & $\mathrm{~S}_{\text {all }}, \mathrm{R}, \mathrm{R}_{c}, \mathrm{~F}_{n}, \mathrm{~F}_{e}$ & $\mathrm{~S}_{\text {all }}, \mathrm{F}_{e}, \mathrm{~F}_{w}$ & $\mathrm{R}_{c c}$ & - & - & - & - \\
\hline RI & $\mathrm{T}, \mathrm{D}, \mathrm{S}_{\text {all }}, \mathrm{F}_{e}, \mathrm{Tr}$ & $\mathrm{D}, \mathrm{S}, \mathrm{S}_{u}$ & $\mathrm{R}$ & & $\mathrm{D}, \mathrm{S}_{\text {all }}, \mathrm{R}_{c c}$ & $\mathrm{~S}_{\text {all }}$ & - & - & - \\
\hline $\mathrm{RM}$ & all but $\mathrm{T}, \mathrm{R}_{c c}$ & $\mathrm{D}, \mathrm{R}_{c}$ & $\mathrm{R}_{c}$ & $\mathrm{D}, \mathrm{R}, \mathrm{R}_{c}, \mathrm{~F}_{e}$ & $\mathrm{D}, \mathrm{S}, \mathrm{S}_{d}, \mathrm{R}_{\text {all }}, \mathrm{F}_{n}$ & $\mathrm{~S}, \mathrm{~S}_{d}, \mathrm{R}, \mathrm{R}_{c}, \mathrm{~F}_{n}$ & $\mathrm{R}, \mathrm{R}_{c}$ & - & - \\
\hline $\mathrm{RR}$ & all but $\mathrm{S}_{d}, \mathrm{R}_{c c}, \mathrm{~F}_{s}, \mathrm{~F}_{n}$ & & $\mathrm{D}$ & $\mathrm{S}_{d}, \mathrm{~F}_{e}$ & $\mathrm{~S}_{\text {all }}, \mathrm{R}_{\text {all }}$ & $\mathrm{S}_{\text {all }}, \mathrm{R}, \mathrm{R}_{c}$ & $\mathrm{R}_{c}$ & $\mathrm{D}$ & - \\
\hline RL & $\mathrm{T}, \mathrm{F}_{e}$ & $\mathrm{~S}, \mathrm{~S}_{d}, \mathrm{R}, \mathrm{R}_{c c}$ & $\mathrm{D}, \mathrm{S}_{a l l}, \mathrm{R}, \mathrm{R}_{c c}, \mathrm{Tr}$ & $\mathrm{T}, \mathrm{S}_{\text {all }}, \mathrm{Tr}$ & $\operatorname{Tr}$ & $\mathrm{D}, \mathrm{F}_{w}$ & $\mathrm{D}, \mathrm{S}_{\text {all }}$ & $\mathrm{D}, \mathrm{S}_{\text {all }}, \mathrm{R}, \mathrm{R}_{c}$ & $\mathrm{~S}_{\text {all }}, \mathrm{R}, \mathrm{R}_{c}$ \\
\hline
\end{tabular}

Table 6: Summary of the post-hoc analysis on Pitch for the FINGER factor. The cell of the table indicates the tasks on which two fingers (first row and first column) differ. All $p<0.05$.

\begin{tabular}{cccccc}
\hline & $\mathrm{T}$ & $\mathrm{D}$ & $\mathrm{S}$ & $\mathrm{R}$ & $\mathrm{F}$ \\
\hline $\mathrm{T}$ & - & - & - & - & - \\
$\mathrm{D}$ & RT, LL & - & - & - & - \\
$\mathrm{S}$ & all fingers & all fingers & - & - & - \\
$\mathrm{R}$ & all fingers & all fingers & all but LI, LT, RI & - & - \\
$\mathrm{F}$ & $\mathrm{RT}$ & $\mathrm{LL}$ & all fingers & all fingers & - \\
$\mathrm{Tr}$ & LI, RT, LL & & all fingers & all fingers & \\
\hline
\end{tabular}

Table 7: Summary of the post-hoc analysis on Roll and Pitch for the TASK factor. The cell of the table indicates the fingers on which two tasks (first row and first column) differ. All $\mathbf{p}<0.04$.

actions without disturbing natural finger orientation during the primary task. Studying Figure 6, we can also observe that the index and middle fingers from both hands are not used with excessive roll angles - except for rotations and scalings - (left: mean roll angle of $-4^{\circ}$ with $95 \%$ CI $\left[-8^{\circ} ;-3^{\circ}\right]$; right: mean roll angle of $12^{\circ}$ with $\left.95 \% \mathrm{CI}\left[9^{\circ} ; 15^{\circ}\right]\right)$. Absolute high roll angles could also be used as another degree of freedom.

Another possible use of angle characterization could help filtering out unwanted contacts. If only contacts starting within the normal range of orientations are processed, it could reduce unintentional interaction with devices that could be due to external factors such as touchscreen repositioning.

In recent years finger identification have been explored to enrich touch expressiveness. Arguably, the most convenient technology to enable it is to recognize fingerprints. Using capacitive fingerprint scanner one can envision an entire capacitive display able to read fingerprints. Sonavation ${ }^{7}$ has

\footnotetext{
${ }_{7 \text { http: //www. sonavation. com/touch-under-glass/ }}$
}

\begin{tabular}{|c|c|c|c|c|c|c|c|c|c|c|}
\hline & $\mathrm{LL}$ & LR & LM & LI & LT & RT & RI & $\mathrm{RM}$ & RR & RL \\
\hline $\mathrm{T}$ & 00 & 000 & 000 & 000 & 000 & 000 & 000 & 000 & $\triangle \circ 0$ & 000 \\
\hline D & ○ & $\nabla \nabla 0$ & $\nabla \nabla 0$ & $\nabla \circ 0$ & $\nabla \nabla 0$ & ००० & $\nabla \circ 0$ & $\nabla \nabla 0$ & $\nabla \nabla 0$ & $\nabla \nabla \circ$ \\
\hline S & $\nabla \nabla \circ$ & $\nabla \nabla 0$ & $\nabla \nabla 0$ & $\nabla \nabla \circ$ & 000 & $0 \Delta \Delta$ & $\nabla \nabla \circ$ & $\nabla \nabla \circ$ & $\nabla \nabla 0$ & $\nabla \circ \circ$ \\
\hline $\mathrm{S}_{d}$ & $\nabla \nabla \circ$ & 000 & $\nabla \nabla 0$ & $\nabla \nabla \circ$ & 000 & $\circ \Delta \Delta$ & $\nabla \nabla \circ$ & $\nabla \circ 0$ & $\nabla \nabla 0$ & $\nabla \circ \Delta$ \\
\hline $\mathrm{S}_{u}$ & 000 & $\nabla \nabla \circ$ & $\nabla \nabla 0$ & $\nabla \nabla \circ$ & $\checkmark \circ 0$ & $\circ \Delta \Delta$ & $\nabla \nabla 0$ & $\nabla \nabla 0$ & $\nabla \nabla 0$ & $\nabla \nabla \circ$ \\
\hline $\mathrm{R}$ & $\nabla \circ 0$ & $\nabla \circ 0$ & $\nabla \nabla 0$ & $\nabla \nabla \circ$ & $\nabla \nabla 0$ & $\nabla \circ \Delta$ & $\nabla \nabla \circ$ & $\nabla \nabla 0$ & $\nabla \nabla$ & $\nabla \nabla \circ$ \\
\hline $\mathbf{R}_{c c}$ & $\nabla \circ \circ$ & $\nabla \nabla \circ$ & $\nabla \nabla 0$ & $\nabla \nabla \circ$ & $\checkmark \circ 0$ & & $\nabla \circ 0$ & $\nabla \nabla 0$ & $\nabla \nabla 0$ & $\nabla \nabla 0$ \\
\hline $\mathrm{R}_{c}$ & $\nabla \circ 0$ & 000 & 000 & $\nabla \circ 0$ & 000 & $\nabla \circ 0$ & $\nabla \nabla \circ$ & $\nabla \nabla 0$ & $\nabla \nabla 0$ & $\nabla \nabla 0$ \\
\hline $\mathrm{F}$ & 000 & 000 & $\circ>$ & $\circ \nabla$ & 000 & $\nabla \nabla 0$ & $\nabla \nabla 0$ & 000 & 000 & $\nabla \nabla 0$ \\
\hline $\mathrm{F}_{S}$ & 000 & 000 & $\circ>0$ & 000 & 000 & 000 & 000 & 000 & 000 & $\nabla \nabla 0$ \\
\hline $\mathrm{F}_{n}$ & $\nabla \nabla 0$ & 000 & 000 & 000 & $\nabla \nabla 0$ & $\nabla \nabla 0$ & $\nabla \nabla \circ$ & $\nabla \nabla 0$ & 000 & $\nabla \nabla \circ$ \\
\hline $\mathrm{F}_{e}$ & 000 & 000 & 000 & 000 & 000 & 000 & 000 & 000 & 000 & 000 \\
\hline $\mathrm{F}_{w}$ & 000 & ००० & ००० & ००० & ००० & $\nabla \nabla \circ$ & ००० & 000 & ००० & 000 \\
\hline $\mathrm{Tr}$ & $\nabla \Delta \Delta$ & 000 & $\nabla \nabla 0$ & $\nabla \circ \Delta$ & 000 & $\triangle \circ O$ & $\circ \wedge$ & $\checkmark \nabla \circ$ & V & $\nabla \nabla$ \\
\hline
\end{tabular}

Table 8: Summary of the post-hoc on Roll and Pitch in the world frame of reference for the different tilt of tablet. $\bigcirc \circ \circ$ correspond to the comparison between tilt $0^{\circ}$ and $15^{\circ}, 0^{\circ}$ and $30^{\circ}$, and $15^{\circ}$ and $30^{\circ}$. When $\circ$ is replaced by $\wedge$ the second tilt yields a bigger area of bounding box, when replaced by $\nabla$ the second tilt yields a smaller area. For instance, $\circ \Delta \nabla$ means that the $30^{\circ}$ bounding box is bigger than the $0^{\circ}$ one and that the $30^{\circ}$ bounding box is smaller than the $15^{\circ}$ one. All $p>0.05$.

patented a touch sensor [31] capable of detecting touch and biometric information such as fingerprints under a glass layer. The best case scenario for fingerprint recognition is when the finger contacts the sensor flat since it is well known this area of the skin carries enough information in minutiae to discriminate individuals and fingers [19]. However it is unclear if non-flat fingers also have enough minutiae to reliably identify them. Using our characterization, we are able to identify which partial part of the fingerprint (i.e. which finger orientation) has to be recognizable in order for such a technology 
to support finger identification in a normal context of use. It seems that from our findings, the side of the finger pad is the key part that has to be recognizable.

Another takeaway from this study is the difference between the thumb roll orientations and those from the other fingers of the same hand. Orientation seems to be a good discriminating information to identify the thumb from the rest of the fingers. Furthermore, unsurprisingly the physical symmetry of our hand is preserved relatively well when interacting. A GUI aware of the roll angle at which fingers interact with it could be used to adapt itself automatically to handedness (for instance, by adapting the position of contextual menus in a table top interaction context). However, differentiating the left hand from the right hand seems only possible if a subset of finger are used: either all fingers but thumbs or just the thumbs.

\section{LIMITATIONS AND FUTURE WORK}

This work characterizes the pitch and roll orientations for all fingers for a flat touchscreen and can be useful for several contexts of interaction (e.g. horizontal tabletops or the use of tablets flat on a desk). However the lack of yaw information reduced the number of factors that could be tested. As measuring natural yaw, pitch and roll finger orientations together remains an open problem, this paper provides only a starting point for the area. An obvious direction for future works is to identify a robust technical solution to measure yaw, pitch, and roll, in a way that does not interfere with the user's natural behaviour. Combining an IMU with the use of state-of-theart yaw recognition (e.g. [37]) is worth investigating, since it is not clear to what extent finger postures are constrained. Our results can provide a baseline for new techniques which seek to measure natural orientations using more cumbersome settings. Nevertheless, our cheap (less than \$60) and easily reproducible setup can be used to investigate other tasks (e.g. typing on a double-touchscreen laptop) or input factors (e.g. position of the screen, such as flat on the lap as identified by [15]).

\section{CONCLUSION}

The performance characteristics of atomic touch interactions, such as tap, drag, scale, rotation and flick, have been extensively studied. However, less is known regarding how they are carried out by users. In this paper we studied the natural pitch and roll orientation of all ten fingers while performing such actions. We used an IMU in a low cost and easily reproducible setup to accurately measure finger orientation. Our results provide a set of baselines about pitch and roll orientation for all the fingers of both hands for one setting (a flat tablet in front of the user). We found that for a given hand, the little, ring and middle fingers are used in a similar manner, whereas the thumb uses different range of orientations. Additional analyses about how changing the angle of the tablet affects people's finger orientations suggest that ranges of orientation tighten as the tablet pitch increases. Our data provides designers and researchers with a new resource to better understand the use of pitch and roll as new degrees of freedom (e.g. using finger pitch as a secondary mode) and to determine whether new sensing techniques are feasible (e.g. using fingerprints for identifying specific finger touches).

\section{REFERENCES}

1. Michelle Annett, Anoop Gupta, and Walter F. Bischof. 2014. Exploring and Understanding Unintended Touch During Direct Pen Interaction. ACM Trans.

Comput.-Hum. Interact. 21, 5, Article 28 (Nov. 2014), 39 pages. DOI : http: //dx. doi.org/10.1145/2674915

2. Myroslav Bachynskyi, Gregorio Palmas, Antti Oulasvirta, Jürgen Steimle, and Tino Weinkauf. 2015. Performance and Ergonomics of Touch Surfaces: A Comparative Study Using Biomechanical Simulation. In Proc. CHI. ACM, 1817-1826.

http://doi.acm.org/10.1145/2702123.2702607

3. Ashley Colley and Jonna Häkkilä. 2014. Exploring Finger Specific Touch Screen Interaction for Mobile Phone User Interfaces. In Proc. OzCHI. ACM, 539-548. http://doi.acm.org/10.1145/2686612.2686699

4. Chi Tai Dang and Elisabeth André. 2011. Usage and Recognition of Finger Orientation for Multi-touch Tabletop Interaction. In Proceedings of the 13th IFIP TC 13 International Conference on Human-computer Interaction - Volume Part III (INTERACT'11). Springer-Verlag, Berlin, Heidelberg, 409-426. http: //dl.acm.org/citation. cfm?id=2042182.2042219

5. Paul M Fitts. 1954. The information capacity of the human motor system in controlling the amplitude of movement. Journal of experimental psychology 47, 6 (1954), 381.

6. Hyunjae Gil, DoYoung Lee, Seunggyu Im, and Ian Oakley. 2017. TriTap: Identifying Finger Touches on Smartwatches. In Proceedings of the 2017 CHI Conference on Human Factors in Computing Systems (CHI '17). ACM, New York, NY, USA, 3879-3890. DOI : http://dx.doi.org/10.1145/3025453.3025561

7. Alix Goguey, Mathieu Nancel, Géry Casiez, and Daniel Vogel. 2016. The Performance and Preference of Different Fingers and Chords for Pointing, Dragging, and Object Transformation. In Proc. CHI'16. ACM, 4250-4261. DOI :

http: //dx.doi.org/10.1145/2858036.2858194

8. Alix Goguey, Daniel Vogel, Fanny Chevalier, Thomas Pietrzak, Nicolas Roussel, and Géry Casiez. 2017. Leveraging finger identification to integrate multi-touch command selection and parameter manipulation. International Journal of Human-Computer Studies 99 (2017), 21 - 36. DOI : http://dx.doi.org/10.1016/j.ijhes.2016.11.002

9. Tovi Grossman, Pierre Dragicevic, and Ravin Balakrishnan. 2007. Strategies for Accelerating On-line Learning of Hotkeys. In Proceedings of the SIGCHI Conference on Human Factors in Computing Systems (CHI '07). ACM, New York, NY, USA, 1591-1600. DOI : http://dx.doi.org/10.1145/1240624.1240865 
10. Carl Gutwin, Andy Cockburn, Joey Scarr, Sylvain Malacria, and Scott C. Olson. 2014. Faster Command Selection on Tablets with FastTap. In Proceedings of the SIGCHI Conference on Human Factors in Computing Systems (CHI'14). ACM, New York, NY, USA, 2617-2626. DOI :

http://dx.doi.org/10.1145/2556288.2557136

11. Anthony D Hall, James B Cunningham, Richard P Roache, and Julie W Cox. 1988. Factors affecting performance using touch-entry systems: Tactual recognition fields and system accuracy. Journal of applied psychology 73, 4 (1988), 711.

12. Eve Hoggan, John Williamson, Antti Oulasvirta, Miguel Nacenta, Per Ola Kristensson, and Anu Lehtiö. 2013. Multi-touch Rotation Gestures: Performance and Ergonomics. In Proceedings of the SIGCHI Conference on Human Factors in Computing Systems (CHI'13). ACM, New York, NY, USA, 3047-3050. DOI : http://dx.doi.org/10.1145/2470654.2481423

13. Christian Holz and Patrick Baudisch. 2010. The Generalized Perceived Input Point Model and How to Double Touch Accuracy by Extracting Fingerprints. In Proceedings of the SIGCHI Conference on Human Factors in Computing Systems (CHI '10). ACM, New York, NY, USA, 581-590. DOI : http://dx.doi.org/10.1145/1753326.1753413

14. Carl J Huberty and Stephen Olejnik. 2006. Applied MANOVA and discriminant analysis. Vol. 498. John Wiley \& Sons.

15. Julien Jacquier-Bret, Philippe Gorce, Genaro Motti Lilian, and Nadine Vigouroux. 2017. Biomechanical analysis of upper limb during the use of touch screen: motion strategies identification. Ergonomics 60, 3 (2017), 358-365. DOI :

http://dx.doi.org/10.1080/00140139.2016.1175671 PMID: 27066760.

16. Sven Kratz, Patrick Chiu, and Maribeth Back. 2013. PointPose: Finger Pose Estimation for Touch Input on Mobile Devices Using a Depth Sensor. In Proceedings of the 2013 ACM International Conference on Interactive Tabletops and Surfaces (ITS '13). ACM, New York, NY, USA, 223-230. DOI : http://dx.doi.org/10.1145/2512349.2512824

17. S. O. H. Madgwick, A. J. L. Harrison, and R. Vaidyanathan. 2011. Estimation of IMU and MARG orientation using a gradient descent algorithm. In 2011 IEEE International Conference on Rehabilitation Robotics. IEEE, 1-7. DOI : http://dx.doi.org/10.1109/ICORR.2011.5975346

18. Sylvain Malacria, Gilles Bailly, Joel Harrison, Andy Cockburn, and Carl Gutwin. 2013. Promoting Hotkey Use Through Rehearsal with ExposeHK. In Proceedings of the SIGCHI Conference on Human Factors in Computing Systems (CHI'13). ACM, New York, NY, USA, 573-582. DOI : http://dx.doi.org/10.1145/2470654.2470735
19. Davide Maltoni, Dario Maio, Anil Jain, and Salil Prabhakar. 2009. Handbook of fingerprint recognition. Springer Science \& Business Media.

20. Nicolai Marquardt, Johannes Kiemer, David Ledo, Sebastian Boring, and Saul Greenberg. 2011. Designing user-, hand-, and handpart-aware tabletop interactions with the TouchID toolkit. In Proc. ITS. ACM, 21-30. http://doi.acm.org/10.1145/2076354.2076358

21. Sven Mayer, Perihan Gad, Katrin Wolf, Paweł W. Woźniak, and Niels Henze. 2017a. Understanding the Ergonomic Constraints in Designing for Touch Surfaces. In Proceedings of the 19th International Conference on Human-Computer Interaction with Mobile Devices and Services (MobileHCI '17). ACM, New York, NY, USA, Article 33, 9 pages. DOI :

http://dx.doi.org/10.1145/3098279.3098537

22. Sven Mayer, Michael Mayer, and Niels Henze. 2017b. Feasibility Analysis of Detecting the Finger Orientation with Depth Cameras. In Proceedings of the 19th International Conference on Human-Computer Interaction with Mobile Devices and Services (MobileHCI '17). ACM, New York, NY, USA, Article 82,8 pages. DOI :

http://dx.doi.org/10.1145/3098279.3122125

23. IC McManus, R_I Kemp, and J Grant. 1986. Differences between fingers and hands in tapping ability: dissociation between speed and regularity. Cortex 22, 3 (1986), 461-473.

24. Lilian Genaro Motti Ader, Nadine Vigouroux, and Philippe Gorce. 2017. Movement Analysis for Improving Older Adults' Performances in HCI: Preliminary Analysis of Movements of the Users' Wrists During Tactile Interaction. Springer International Publishing, Cham, 17-26. DOI :

http://dx.doi.org/10.1007/978-3-319-58536-9_2

25. Alexander Ng, Stephen A. Brewster, and John H. Williamson. 2014. Investigating the Effects of Encumbrance on One- and Two- Handed Interactions with Mobile Devices. In Proceedings of the SIGCHI Conference on Human Factors in Computing Systems (CHI '14). ACM, New York, NY, USA, 1981-1990.

DOI : http://dx.doi.org/10.1145/2556288.2557312

26. Esben Warming Pedersen and Kasper Hornbæk. 2012. An Experimental Comparison of Touch Interaction on Vertical and Horizontal Surfaces. In Proceedings of the 7th Nordic Conference on Human-Computer Interaction: Making Sense Through Design (NordiCHI '12). ACM, New York, NY, USA, 370-379. DOI : http://dx.doi.org/10.1145/2399016.2399074

27. Simon Rogers, John Williamson, Craig Stewart, and Roderick Murray-Smith. 2011. AnglePose: Robust, Precise Capacitive Touch Tracking via 3D Orientation Estimation. In Proceedings of the SIGCHI Conference on Human Factors in Computing Systems (CHI'11). ACM, New York, NY, USA, 2575-2584. DOI : http: //dx.doi.org/10.1145/1978942.1979318 
28. Q. Roy, Y. Guiard, G. Bailly, E. Lecolinet, and O. Rioul. 2015. Glass+Skin: An Empirical Evaluation of the Added Value of Finger Identification to Basic Single-Touch Interaction on Touch Screens. In INTERACT'15: IFIP International Conference on Human-Computer Interaction. Springer, Bamberg, Germany.

29. I. Scott MacKenzie. 2015. Fitts' Throughput and the Remarkable Case of Touch-Based Target Selection. In Human-Computer Interaction: Interaction Technologies (Lecture Notes in Computer Science), Masaaki Kurosu (Ed.), Vol. 9170. Springer International Publishing, 238-249. DOI : http://dx.doi.org/10.1007/978-3-319-20916-6_23

30. Andrew Sears and Ben Shneiderman. 1991. High precision touchscreens: design strategies and comparisons with a mouse. International Journal of Man-Machine Studies 34, 4 (1991), 593-613.

31. Dale R Setlak, John C Schmitt, Steven D Wilson, and Ellery Y Chan. 1998. Fingerprint sensing apparatus with finger position indication. (Dec. 22 1998). US Patent $5,852,670$

32. Atsushi Sugiura and Yoshiyuki Koseki. 1998. A user interface using fingerprint recognition: holding commands and data objects on fingers. In Proc. UIST. ACM, 71-79. DOI :

http://dx.doi.org/10.1145/288392.288575

33. Feng Wang, Xiang Cao, Xiangshi Ren, and Pourang Irani. 2009. Detecting and leveraging finger orientation for interaction with direct-touch surfaces. In Proc. UIST.
ACM, 23-32. DOI :

http://dx.doi.org/10.1145/1622176.1622182

34. Feng Wang and Xiangshi Ren. 2009a. Empirical Evaluation for Finger Input Properties in Multi-touch Interaction. In Proc. CHI. ACM, 1063-1072. http://doi .acm.org/10.1145/1518701.1518864

35. Feng Wang and Xiangshi Ren. 2009b. Empirical Evaluation for Finger Input Properties in Multi-touch Interaction. In Proceedings of the SIGCHI Conference on Human Factors in Computing Systems (CHI '09). ACM, New York, NY, USA, 1063-1072. DOI : http://dx.doi.org/10.1145/1518701.1518864

36. Yoichi Watanabe, Yasutoshi Makino, Katsunari Sato, and Takashi Maeno. 2012. Contact Force and Finger Angles Estimation for Touch Panel by Detecting Transmitted Light on Fingernail. Springer Berlin Heidelberg, Berlin, Heidelberg, 601-612. DOI : http://dx.doi.org/10.1007/978-3-642-31401-8_53

37. Robert Xiao, Julia Schwarz, and Chris Harrison. 2015. Estimating 3D Finger Angle on Commodity Touchscreens. In Proc ITS. ACM, 47-50. DOI : http://dx.doi.org/10.1145/2817721.2817737

38. Hong Zhang, Xing-Dong Yang, Barrett Ens, Hai-Ning Liang, Pierre Boulanger, and Pourang Irani. 2012. See Me, See You: A Lightweight Method for Discriminating User Touches on Tabletop Displays. In Proceedings of the SIGCHI Conference on Human Factors in Computing Systems (CHI '12). ACM, New York, NY, USA, 2327-2336. DOI : http://dx.doi.org/10.1145/2207676.2208392 